\title{
Franziska Lang, Andreas Sidler (Hrsg.): Psychodynamische Organisationsanalyse und Beratung. Einblicke in eine neue Disziplin (Psychosozial-Verlag, Giessen 2007)
}

Sonja Wuhrmann (Zürich)

Auch wenn eine Kritik an den Anfang gestellt wird, so soll doch vorweggenommen werden, dass dieses Buch einen wertvollen Überblick über die unterschiedlichen Vorgehensweisen in der Organisationsberatung verschafft, die auf die Psychoanalyse rekurrieren. Die Kritik gilt dem Untertitel «Einblicke in eine neue Disziplin». Es ist schon erstaunlich, wie wenig offenbar wahrgenommen wird, was sich aus der Psychoanalyse heraus entwickelt hat. So kann das Tavistock Institute in London immerhin auf eine 50-jährige Geschichte im Bereich der Organisationsberatung zurückblicken. Wenn wir davon ausgehen, dass auch eine Titelgebung einen unbewussten Anteil beinhaltet, so kommt darin tatsächlich ein Defizit zum Ausdruck. In der Einleitung schreiben die Autorin und der Autor nämlich, dass die Breite der Texte auch eine Vielfalt an unterschiedlich verwendeten Begriffen und Konzepten beinhaltet, die sichtbar macht, dass eine kongruente und systematische Theoriebildung der psychodynamischen Beratungsmethode noch fehlt, ja dass es die psychodynamische Beratungsmethode so (noch) gar nicht gibt. Allen psychoanalytischen Ansätzen gemeinsam ist aber, dass ein minimal strukturiertes Setting im Zentrum steht, welches es den Mitgliedern einer Organisation ermöglicht, in freier Aussprache ihr Erleben in der Organisation zu reflektieren. Dabei werden abgewehrte oder latente Konflikte bewusst und können durch Bearbeitung aufgelöst werden. Berücksichtigt man, dass heute eine Organisation charakterisiert ist durch ständige Veränderungsprozesse, die Widerstände nach sich ziehen, so wird deutlich, wie hilfreich eine Methode sein kann, deren Ziel es ist, gerade solche Widerstände bewusst zu machen. Dies führt zur Entlastung der Mitarbeitenden, zur Integration des Neuen und trägt damit zur Entwicklung der Organisation bei. Oder wie Beate West-Leuer in ihrem Artikel betont, oberstes Ziel einer psychodynamischen Beratung im Einzelcoaching sei es, dass sie Hilfe zur Selbsthilfe böte. Das gilt insgesamt für die psychoanalytische Beratung, ob sie sich nun auf ein Einzelcoaching, eine Teamsupervision oder auf die Beratung der Gesamtorganisation bezieht. 
Das Buch ist aus dem Vortragszyklus «Psychoanalyse - Organisation Beratung» im Wintersemester 2005/2006 am Psychoanalytischen Seminar Zürich hervorgegangen. Die sechs Vortragenden: Kurt Buchinger, Rolf Haubl, Olaya Khaleelee, Ross A. Lazar, Burkhard Sievers und Beate West-Leuer sind bedeutende VetreterInnen dieses Bereiches, und es ist Franziska Lang und Andreas Sidler zu gratulieren, dass sie es geschafft haben, mit diesem Zyklus auch ein Stück Geschichte der psychoanalytischen Organisationsberatung nach Zürich zu bringen.

Die Beiträge «Learning from the Inside Out: Gruppenbeziehungen und Organisationsberatung» von Olaya Khaleelee und «Führung schaffen Folgende schaffen Führende» von Ross A. Lazar ergänzen sich wohl auch deshalb sehr gut, weil beide dem Group Relations-Ansatz des Tavistock Institute in London verpflichtet sind. Diese Beiträge vermitteln Wesentliches zur Geschichte und Entwicklung der psychoanalytischen Arbeit in Organisationen. So wird die Geschichte des Group Relations-Ansatzes bis auf seine Anfänge, 1946, beschrieben. Bedeutsam dabei und zur Grundlagenliteratur in der psychodynamischen Organisationsberatung gehörend ist die Theorie der Grundannahmegruppen von Wilfred R. Bion. Die Unterscheidung zwischen Arbeitsgruppe und Grundannahmegruppe ist äusserst hilfreich, wenn es darum geht, irrationales Verhalten in Gruppen oder Teams zu verstehen. Eine Gruppe verfügt über zwei Verhaltensweisen, über ein rationales, sachbezogenes Arbeitsverhalten (die Arbeitsgruppe) und ein Verhalten, das durch die Dynamik bestimmt wird bzw. über den Grad der Angst, der die Gruppe beherrscht. Kommt eine Gruppe zusammen, so sind nämlich die Einzelnen unbewusst auf deren Erhalt bezogen und bekämpfen die Angst vor Zerfall mit passivem oder aggressivem Verhalten, was Bion die Grundannahmegruppen genannt hat. (Die drei Grundannahmen sind Abhängigkeit, Kampf/Flucht und Paarbildung). Sind die Grundannahmen aktiv, so kann die «primäre Aufgabe» nur noch schlecht oder gar nicht mehr erfüllt werden. Die «primäre Aufgabe» - ebenfalls ein Konzept aus der Tavistock Tradition - bezeichnet den Auftrag oder die Aufgabe, die eine Gruppe von Mitarbeitenden auszuführen hat. Kann sich eine Gruppe der «primären Aufgabe» widmen, ihren Auftrag arbeitsbezogen und sachlich erfüllen, so spricht man von einer Arbeitsgruppe. Zwar sind die Grundannahmen immer latent aktiv, sie dominieren aber nicht über das rationale Arbeitsverhalten. Ross A. Lazar fokussiert in seinem Beitrag denn auch auf die Gruppe. Nachdem sich die Literatur hauptsächlich auf die Bedeutung der Führung konzentriert, und so im Grunde immer die Vorstellung eines Top down zementiert wird, wird die Bedeutung der Folgschaft, also die Mitarbeitenden, vernachlässigt. Die Kultur einer Organisation, so die implizite Vorstellung, wird immer durch das Oben bestimmt. Lazar bezieht 
sich auf die These von Robert E. Kelley, dass Menschen nicht irgend jemandem folgen, sondern dass es Gründe geben muss, warum sie Folgschaft leisten. Er vertritt die These, dass die Folgschaft Führende schafft. Also genau umgekehrt, Bottom up, wird die Kultur einer Organisation geschaffen. Denkt man z. B. an den Streik der SBB-Angestellten in Bellinzona in diesem Frühling, so ist dies sicher ein Beispiel dafür, wie gross die Macht einer Gruppe bzw. der Folgschaft sein kann. Auch andere politische Beispiele von Folgschaft drängen sich selbstverständlich in diesem Zusammenhang auf. Dies scheint mir gerade heute ein spannender Ansatz zu sein, weil die gegenseitige Beeinflussung von Folgschaft auf Führung und umgekehrt deutlich wird.

Ist die eben geschilderte Sicht eine theoretische, die auch Gesellschaft und Geschichte berücksichtigt, so stellt Beate West-Leuer ein Beratungskonzept für psychodynamisch-systemisches Einzelcoaching vor. Obwohl sie das Gegenteil betont, scheint sich dieses Konzept von einem zielorientierten Denken nicht frei machen zu können. Die durch strukturiertes Vorgehen auflösbaren Konflikte, die dem Coachee zu einem integrativen Führungsstil verhelfen, verführen zu Omnipotenzfantasien, welche sie auch beschreibt. Das Vorgehen beinhaltet sieben Phasen: Kontakt, Annäherung, Diagnose bzw. Abhängigkeiten, Interventionsplanung und Prognose, Begegnung bzw. intersubjektive Reflexion, Beratungsbeziehung bzw. Intervention, Ablösung. Obwohl dieses Konzept von einerWechselwirkung zwischen Person und Organisation ausgeht, wird das Problem des Coachee letztlich auf einem innerpsychischen Hintergrund verstanden und entsprechend bearbeitet. Dieses CoachingKonzept erinnert eher an eine Fokaltherapie. Im Grunde genommen geht es davon aus, dass sich unbewältigte ödipale Konflikte in der Organisation reinszenieren und so zu Konflikten führen. So wird aber die Organisationsdynamik auf die Dyade reduziert und werden Konflikte personalisiert. Die Gefahr dieses Konzeptes sehe ich darin, dass es nicht zum Coaching führt, sondern zur Therapie verführt.

Nicht ein Konzept, sondern Orientierungshilfe will der Beitrag von Rolf Haubl leisten: «Die Unterwelt bewegen ... Zum Selbstverständnis einer psychoanalytischen bzw. gruppenanalytischen Organisationsanalyse und Organisationsberatung.» Einer seiner zentralen Punkte für BeraterInnen mit psychoanalytischem oder gruppenanalytischem Hintergrund betrifft die kulturell und gesellschaftlich geprägten Organisationsmetaphern. In den letzten Jahren ist das hauptsächlich die Vorstellung der Organisation als einer Kultur. Die psychoanalytische Sozialpsychologie verfügt über einen eigenen Kulturbegriff, dessen Mittelpunkt die Produktion von Unbewusstheit (Erdheim) darstellt. Die Produktion von Unbewusstheit stellt denn auch einen zentralen Aspekt psychoanalytischer 
und gruppenanalytischer Beratung dar. So muss der psychosozialen Abwehr, der Abwehr der Gruppe, hohe Aufmerksamkeit geschenkt werden. Der Latenzschutz, ein bestimmtes Mass an Unbewusstheit, muss aufrecht erhalten werden, damit die Mitarbeitenden ihre primäre Aufgabe wieder erfüllen können. Prädestiniert dafür sind wir als PsychoanalytikerInnen und GruppenanalytikerInnen, weil wir zum Verständnis unbewusster Prozesse das wichtigste Instrument zurVerfügung haben, nämlich mit Übertragung und Gegenübertragung zu arbeiten, uns als Container zur Verfügung zu stellen und nur das zu formulieren, was erträglich scheint und aufgenommen werden kann.

Ich habe mich auf diejenigen Beiträge beschränkt, die mich speziell zum Denken angeregt oderWidersprüche in mir ausgelöst haben, wobei es mir wichtig ist zu betonen, dass alle von hohem Interesse sind. Zwar steht eine Konzeptualisierung der psychodynamischen Methode noch aus, was ein Defizit sein mag, welches aber gleichzeitig einen Freiraum ermöglicht, der noch nicht von dogmatischen Vorstellungen geprägt ist und das eigene Denken fördert. 